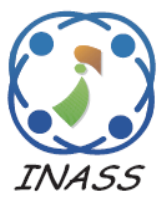

\title{
Cyclostationary Feature Detection Scheme for FBMC and OFDM Cognitive Radio Systems
}

\author{
Mohammed K. Al-Haddad ${ }^{1 *}$ \\ Hadi T. Ziboon ${ }^{2}$ \\ ${ }^{I}$ Electronics and Communications Engineering Department, University of Baghdad, Iraq \\ ${ }^{2}$ Electrical Engineering Department, University of Technology, Iraq \\ *mohammed.alhadad@coeng.uobaghdad.edu.iq
}

\begin{abstract}
Due to the inefficient use of the licensed frequency spectrum, a significant portion of the spectrum is left unutilized. This has led to the emerging of the Cognitive Radio (CR) as new technology that can access the white spaces in the licensed spectrum for improved spectrum utilization efficiency. In this paper, we present a scheme for detecting cyclostationary feature embedded in multicarrier signals like Orthogonal Frequency Division Multiplexing (OFDM) and Filter Bank Multicarrier (FBMC). The cyclostationary feature is generated due to the presence of pilot signals at certain subcarriers commonly used for channel estimation and frequency offset estimation. Therefore, no especial restrictions on the Primary User (PU) signal are imposed in order to generate the desired Cyclostationary Signature (CS). Both cases of scattered pilots and continuous pilots are considered. The algorithm is based on observing a periodic pattern in the cyclic autocorrelation function created by the pilot subcarriers, this periodic pattern is considered the cyclic signature and it can be extracted without prior knowledge of the signal parameters. The performance is evaluated under different impairments conditions like timing offset, frequency offset and multipath channel in addition to AWGN. Results show that FBMC has about 2-3dB signal-to-noise ration advantage over OFDM in continuous pilots mode and both modulations perform comparably in scattered pilots mode.
\end{abstract}

Keywords: Cognitive radio, OFDM, FBMC, Cyclic autocorrelation, Cyclostationary signature.

\section{Introduction}

The increasing demand for wireless communications and the scarcity of the spectrum resources have motivated the research for alternative solutions like Cognitive Radio (CR). In CR, secondary user (SU) can have access to white spaces in the licensed spectrum created by the absence of the Primary User (PU). This dynamic spectrum utilization can improve the spectral efficiency of the overall system $[1,2]$. The term CR refers to the adaptation of the communication system parameters including operating frequency. Spectrum Sensing (SS) CR system is a communications system that particularly adapts the operating frequency to the detected white spaces in the spectrum. There are different SS techniques that vary in terms of performance and complexity. The most common technique is energy detection [3], which is considered the simplest technique, however, it can only determine if a signal is present but cannot determine the type of signal also it requires the estimate of the noise variance that leads to the problem of noise uncertainty. The matched filter technique [4] has better performance at low Signal-to-Noise Ratio (SNR) but it requires prior knowledge of the PU signal parameters in order to make synchronized signal detection and this can lead to high complexity. Cyclostationary feature detection technique [2] can perform well for low SNR and it is suitable for multicarrier modulation (MCM) signals; the complexity is higher than the energy detectors but can be considered moderate. These techniques fall under the general category of non-cooperative CR and they operate at the physical layer of a communication system. On the other hand, there are techniques of cooperative $\mathrm{CR}$ that operate on a network level like interweave, underlay and overlay CR [5]. Recently, 
the cyclostationary feature detection has attracted wider attention in CR applications because of its ability to overcome the shortcomings of the conventional energy detection methods. Noise is a stationary process because its autocorrelation is time independent, a modulated signal is a cyclostationary process meaning its autocorrelation is periodically time dependent. This sets a clear distinction between the hypothesis of the absence of the PU $\left(\mathrm{H}_{0}\right)$ and the hypothesis of presence of $\mathrm{PU}\left(\mathrm{H}_{1}\right)$. MCM signals like Orthogonal Frequency Division Multiplexing (OFDM) and Filter Bank Multicarrier (FBMC) have weak inherent cyclostationary properties [6,7], however, strong cyclostationary signature (CS) can be induced in such signals by mapping a set subcarriers to a different set of subcarriers as suggested by [6] and further developed by [7]. This technique imposes certain requirement on the PU transmitter in order for the CR to perform the cyclostationary-based detection. In order to overcome the multipath channel effect, more sets of subcarriers are mapped to different sets of subcarriers as suggested by [8] but this approach increases the overhead of the mapped subcarriers. In some scenarios, certain cyclostationary signatures are intentionally induced in the OFDM signal to distinguish between different OFDM systems [2]. In [9], the CS is induced by pilot signals, the detection of CS is based on analysis of the cyclic spectrum at certain values of cyclic frequency called feature points; a prior knowledge of these feature points reduce the two dimensional cyclic spectrum to one dimension. Pilot induced CS is also investigated for OFDM signals in [10]. A cyclic prefix (CP) induced CS detection is proposed in [11] with an alternative configuration of having filter to replace the $\mathrm{CP}$ at the transmitter and receiver, the filter utilizes the bandwidth wasted by the CP; a CP assisted sensing is also presented in [12]. In [13, 14] a maximum cyclic autocorrelation selection (MCAS) technique is investigated to detect the CS signature induced by the $\mathrm{CP}$, in this technique the problem of noise uncertainty is avoided. However, in order to control the strength of the CS induced by the $\mathrm{CP}$, the length of the $\mathrm{CP}$ needs to be manipulated but $\mathrm{CP}$ length is a key parameter in the overall performance of a communication system such approach may not be suitable [6] especially when the CR performance enhancement requires relatively high $\mathrm{CP}$ length as in [13]. In addition, FBMC does not have a CP and it is a candidate for future communications, so, it is advantageous to adopt a more practical CS inducing alternative like pilot subcarriers. In [15] The CS detection approach is investigated for detection of CDMA signals and in [16] the CS detection is applied to single carrier modulation signals. In [17] autocorrelation based technique is applied to FBMC signal, the detected feature is introduced after post processing of the received FBMC signal. In summary, the cyclostationary-based SS can be decomposed into two points; the first is how to introduce the CS in the PU signal and the second is how to detect this CS. In this paper, we adopt the pilot based technique for inducing the CS in the PU transmitted signal with pilots scattered in time or continuous in time. This requirement is already fulfilled in practical systems as pilot signals used for channel estimation and carrier frequency offset estimation, so, there are no special conditions imposed on the PU signal. The metric for detecting the CS is obtained from the cyclic autocorrelation function at zero time lag making it a function of the cyclic frequency only. A pattern of periodic impulses is introduced at the proposed metric that can be detected by correlation with a reference pattern of impulses. This has the advantage of having the detection to be achieved irrespective of the noise variance. Unlike the previous works as in $[2,3,6-9,11,13]$ where the CS is induced at a certain cyclic frequency and the main calculations are performed at that specific value of cyclic frequency, this requires prior knowledge of the signal parameters in order to find this specific cyclic frequency. Otherwise, the main calculations have to be performed for different values of cyclic frequency and hence significantly increasing the complexity. Another advantage of our proposed method is that it is robust against symbol timing and carrier frequency offset.

The demand for higher data rate is increasingly shifting the attention towards MCM especially in wireless communication applications. CP-OFDM is the most common form of MCM used in communications systems like Long Term Evolution (LTE). However, recently the attention is moving towards FBMC as a new MCM technique [18]. FBMC employs localized pulse shaping instead of the basic rectangular pulse shape used in OFDM. The pulse shaping in FBMC leads to improved spectral shaping and can combat the effect of multipath channel without the need for $\mathrm{CP}$, hence improving the spectral efficiency of FBMC over CP-OFDM [19]. The advantages of FBMC over CP-OFDM come on the expense of increased complexity due to the shaping filter and the symbol overlap because the shaping filter duration is greater than the symbol transmission time. Another aspect that makes FBMC different from OFDM is that the transmitted symbols in FBMC are not complex Quadrature Amplitude Modulation (QAM) symbols; instead, the symbols are transmitted in Offset QAM (OQAM) where the 
real and imaginary parts of the QAM symbols are transmitted successively over a half symbol period [20-22].

The rest of the paper is organised as follows. In Section 2, a theoretical background is presented describing the basics of the cyclostationary analysis and the main approach used in literature to induce and detect the CS. In Section3, the proposed method for inducing and detecting the CS is described. In Section 4 , the performance of the proposed scheme is evaluated by numerical simulations. In section 5 , conclusions and final discussion.

\section{Theoretical background}

In OFDM transmission, complex QAM data symbols $c_{n, k}=a_{n, k}+j b_{n, k}$ modulates the $k^{\text {th }}$ subcarrier at the $n^{\text {th }}$ symbol interval. The expression for the CPOFDM signal is given by

$$
x(t)=\sum_{n=-\infty}^{\infty} \sum_{k=0}^{N-1} c_{n, k} \operatorname{rect}\left(t-n\left(T_{0}+T_{1}\right)\right) e^{j 2 \pi k t / T_{0}}
$$

Where $\operatorname{rect}(t)$ is the basic rectangular pulse, $T_{0}$ is the symbol interval and $T_{1}$ is the CP duration. While in FBMC case, OQAM transmission is used where the real part and imaginary part of $c_{n, k}$ are transmitted with $T_{0} / 2$ delay. The FBMC signal can be expressed as

$$
\begin{gathered}
x(t)=\sum_{n=-\infty}^{\infty} \sum_{k=0}^{N-1} a_{n, k} g\left(t-n T_{0}\right) j^{k} e^{j 2 \pi k t / T_{0}}+ \\
\sum_{n=-\infty}^{\infty} \sum_{k=0}^{N-1} j b_{n, k} g\left(t-n T_{0}-T_{0} / 2\right) j^{k} e^{j 2 \pi k t / T_{0}}
\end{gathered}
$$

Where $g(t)$ is the pulse shaping filter with duration of $K T_{0} ; K$ is called the overlapping factor. The shaping filter should satisfy certain conditions of locality and orthogonality discussed in $[20,22]$. The term $j^{k}$ contributes to the orthogonality of the FBMC symbols.

The autocorrelation function of a signal $x(t)$ is

$$
R_{x}(t, \tau)=E\left[x(t+\tau / 2) x^{*}(t-\tau / 2)\right]
$$

Where $E[\cdot]$ is the expected value operator and $x^{*}$ is the complex conjugate of $x$. A signal $x(t)$ is defined as a wide sense cyclostationary if $R_{x}(t, \tau)$ is periodic in $t$ with period $T_{0}$ for each time lag $\tau$. Then $R_{x}(t, \tau)$ can be represented by the Fourier series

$$
R_{x}(t, \tau)=\sum_{\alpha} R_{x}^{\alpha}(\tau) e^{j 2 \pi \alpha t}
$$

Where $\alpha=m / T_{0}$ and $m$ is an integer. The Fourier coefficients term $R_{x}^{\alpha}(\tau)$ is the cyclic autocorrelation function (CAF) and it is given by

$$
R_{x}^{\alpha}(\tau)=\lim _{T \rightarrow \infty} \frac{1}{T} \int_{-T / 2}^{T / 2} R_{x}(t, \tau) e^{-j 2 \pi \alpha t} d t
$$

The Fourier transform (FT) of $R_{x}^{\alpha}(\tau)$ with respect to the variable $\tau$ is called the cyclic spectrum function (SCF) of $x(t)$ and it is given by

$$
S_{x}^{\alpha}(f)=\int_{-\infty}^{\infty} R_{x}^{\alpha}(\tau) e^{j 2 \pi f \tau} d \tau
$$

Where $\alpha$ and $f$ are called the cyclic frequency and the spectral frequency respectively. Cyclostationary signature can be imposed on $R_{x}^{\alpha}(\tau)$ and consequently on $S_{x}^{\alpha}(f)$ by duplicating a set of data symbols subcarriers to another set subcarrier indices as suggested by [6]

$$
c_{n, k}=c_{n, k+q} \quad k \in \psi
$$

Where $\psi$ is the set of the mapped subcarriers and $q$ is an integer. In this technique the CS appears as a peak over the spectral frequency $f$ at a discrete value of the cyclostationary frequency $\alpha_{s}=m_{s} / T_{0}$ called the signature cyclic frequency. The cyclic spectrum of $x(t)$ is estimated by

$$
\hat{S}_{x}^{\alpha}(k)=\frac{1}{L} \sum_{l=0}^{L-1} X_{l}(k) X_{l}^{*}(k-\alpha) W(k)
$$

Where $W(k)$ is a spectral smoothing window, $\mathrm{X}_{l}(k)$ is the FT of the $l^{\text {th }} \mathrm{N}$-samples frame of the signal $x(t)$ sampled at $t=T_{0} / N$. and $L$ is the number of frames.

$$
X_{l}(k)=\sum_{n=0}^{N} x(n+l D) e^{-j 2 \pi \imath k / N}
$$

Where $D$ is the overlapping factor between successive frames of $x(n)$. The CS metric is constructed as the peak output to a sliding window filter to $\hat{S}_{x}^{\alpha}(k)$ which can be expressed as

$$
y_{\alpha}=\max _{m} \sum_{k=m}^{K+m} \hat{S}_{x}^{\alpha}(k)
$$


Note that in Eq. (8), the cyclic spectrum is calculated for a specific cyclic frequency $\alpha$. The value $\alpha$ is dictated by the parameters of the PU signal $x(t)$ like subcarriers duplications as in Eq. (7), CP or pilot subcarriers. Lack of knowledge of these parameters requires estimating the cyclic spectrum $\hat{S}_{x}^{\alpha}(k)$ in Eq. (8) at different values of $\alpha$ causing a significant increase in the computational complexity. In the next section, we propose a metric that exhibits a CS and its estimate is independent of the PU parameters.

\section{The proposed scheme for CS generation and detection}

The CS generation proposed by [6] in Eq. (7) is an awkward restriction to the PU communication system in which the spectral efficiency is sacrificed to support a CR system. The reliance on the CP to generate the CS is also undesirable because the strength of the CS depends on the length of the CP that is governed by the PU communication system to balance between the performance and spectral efficiency. In this work, we will consider a more realistic scheme for CS generation. In every communication system there are pilot signals inserted within the data symbols subcarriers. We assume that the subcarrier pilots are assigned fixed values in periodic pattern in time and frequency indices.

$$
c_{n_{0}+l p, k_{0}+m q}=a \quad l, m=0,1,2, \ldots
$$

Where $p$ and $q$ are fixed integers values and $a$ is the assigned pilot value. Fig. 1 shows an example of scattered pilot assignment scheme. The pilots can be continuous in time if $p=1$.

Fig. 2 shows the CAF of both OFDM signal and FBMC signal with CS generated by Eq. (11). The CS is the pattern of small waves around the center peak of the CAF. In the proposed CS detection scheme, we will consider the values of the $\mathrm{CAF}$ at $\tau=0$ i.e. $R_{x}^{\alpha}(0)$; from Eq. (3) and Eq. (5) this can be expressed as

$$
\begin{aligned}
& R_{x}^{\alpha}(0)=\frac{1}{T} \int_{-T / 2}^{T / 2} E\left[|x(t)|^{2}\right] e^{-j 2 \pi \alpha t} d t \\
& =\frac{1}{T} E\left[\int_{-T / 2}^{T / 2}|x(t)|^{2} e^{-j 2 \pi \alpha t} d t\right]
\end{aligned}
$$

In Eq. (12), $R_{x}^{\alpha}(0)$ is the averaging of the FT of $|x(t)|^{2}$ and it can be estimated by averaging the FT of

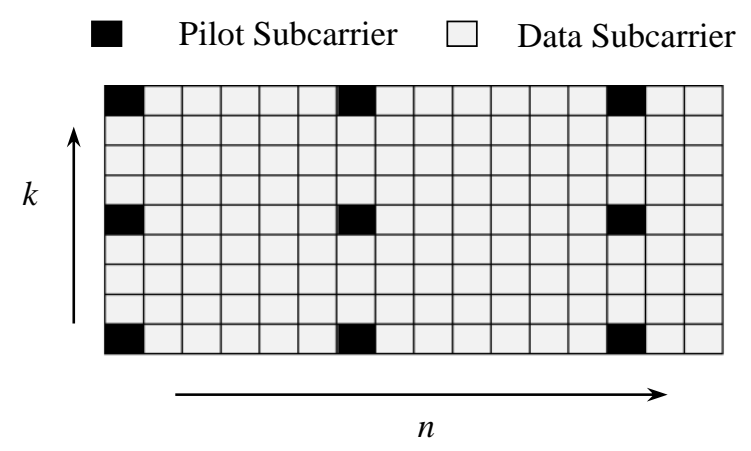

Figure. 1 Example of scattered pilot mapping, $p=6, q=4$, $n_{0}=k_{0}=0$

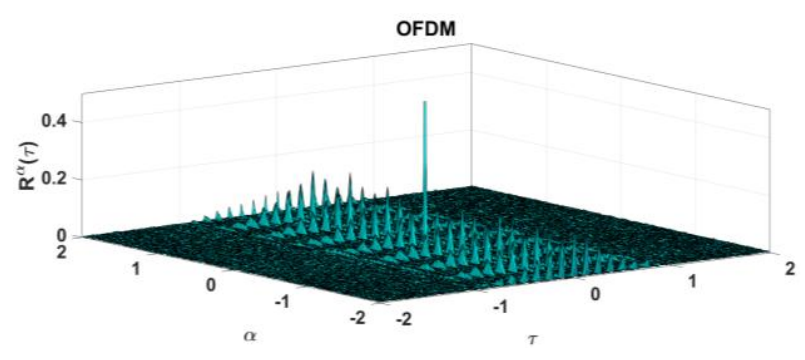

(a)

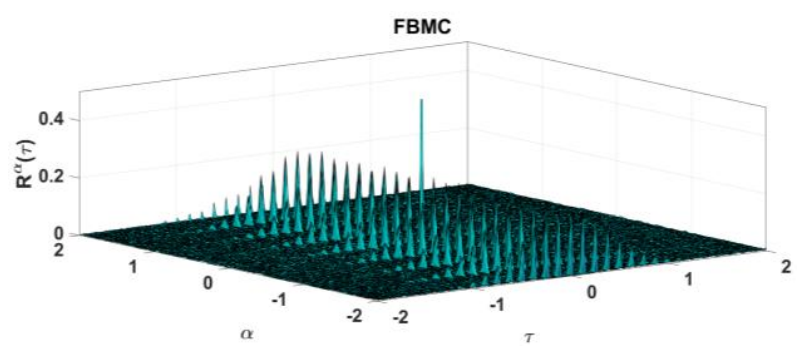

(b)

Figure. 2 The CAF of: (a) OFDM and (b) FBMC

different segments of $x(t)$. The expression in Eq. (12) quite resembles the expression of the Power Spectral Density (PSD) of $|x(t)|^{2}$ where the expected value is evaluated for the magnitude squared of the FT. This alternative is more convenient in the case of timing offset because it removes the phase of the FT of the different segments being averaged that may cause destructive addition. Accordingly, the metric for CS detection is the PSD of $|x(n)|^{2}$ and we will use Welch method for the PSD estimation [23], hence

$$
\begin{aligned}
& y(m)=\frac{1}{L} \sum_{l=0}^{L-1}\left|X_{l}(m)\right|^{2} \\
& X_{l}(m)=\sum_{n=0}^{M-1}|x(n+l D)|^{2} W(n) e^{-j 2 \pi n n}
\end{aligned}
$$

Where $y(m)$ is the metric for CS detection, $D$ is the overlap factor, $W(n)$ is the Hamming window. 
The number of the Fast Fourier Transform (FFT) points, $M$, is a multiple of $N$ and it is chosen to equal the FBMC shaping filter length, i.e., $M=K N$. Since (14) is the FFT of a real function, it is calculated for $0 \leq m \leq M / 2$ due to symmetry. The PU received signal $x(n)$ can be expressed in terms of the transmitted signal $\bar{x}(n)$ and the channel impairments as

$$
x(n)=\sum_{i=0}^{N_{c}-1} h_{i} \bar{x}\left(n_{0}+n+n_{i}\right) e^{j 2 \pi f_{o} n / N}+w(n)
$$

Where $n_{\mathrm{o}}$ offset is the timing and $f_{o}$ is the frequency offset normalized by the subcarrier bandwidth $F$; both $n_{o}$ and $f_{o}$ are assumed unknown to the $\mathrm{CR}$ receiver which is the natural case due to lack of synchronization between the CR receiver and the PU signal. The coefficient and delay of the $i^{\text {th }}$ path of the multipath channel are $h_{i}$ and $n_{i}$ respectively, $N_{c}$ is the number of channel coefficients and $w(n)$ is the Additive White Gaussian Noise (AWGN). For the case of AWGN channel, we set $N_{c}=1$ and $h_{0}=1$.

The metric $y(m)$ is shown Fig. 3 for OFDM, FBMC and noise, the CS generated by Eq. (11) and can be seen as periodic impulses over the cyclic frequency index $m$ while there is no periodic pattern in $y(m)$ in the noise case. The periodicity of the impulses is related to the repetition rate of pilots in frequency which is given by the parameter $q$ in Eq. (11). A decision metric can be calculated as a correlation with a reference pattern of periodic impulses $Y(m)$ of period $q K$, where $K=M / N$, as shown in Fig. 4, i.e.,

$$
\begin{aligned}
& S=\frac{1}{N_{p}} \sum_{k=1}^{N_{p}} y(n) Y(m) \\
& N_{p}=\lfloor M /(4 q K)\rfloor
\end{aligned}
$$

Where $\lfloor x\rfloor$ is the floor of $x, N_{p}$ is the number of impulses used in calculating the decision metric $S$; the impulses of $y(m)$ for $1<m<M / 4$ are only considered because beyond that the impulses will decrease in magnitude.

Eq. (16) can be written as

$$
S=\frac{1}{N_{p}} \sum_{k=1}^{N_{p}} y(k q K)
$$

The probability of detection will be $P_{d}=\operatorname{Pr}\left(S<T h \mid \mathrm{H}_{1}\right)$ and the probability of false alarm $P_{f}=\operatorname{Pr}\left(S<T h \mid \mathrm{H}_{0}\right)$, where $T h$ is the decision threshold.

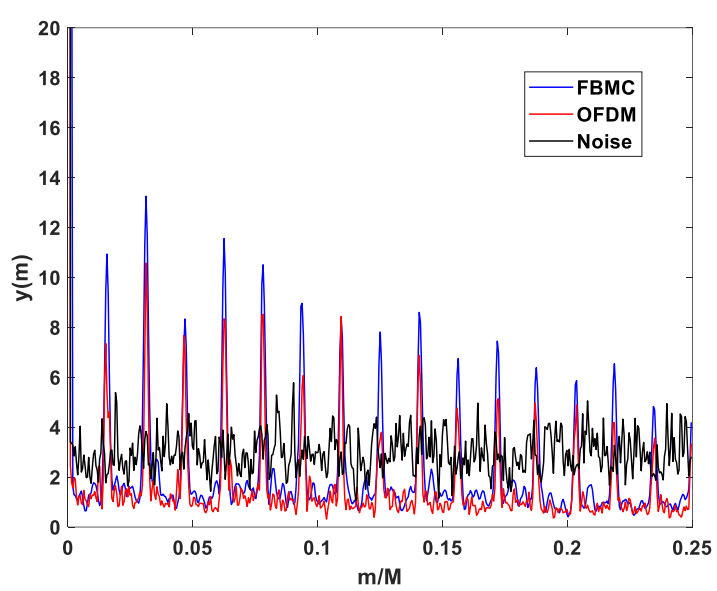

Figure. 3 The metric $y(m)$ with CS as periodic pattern of impulses in FBMC and OFDM

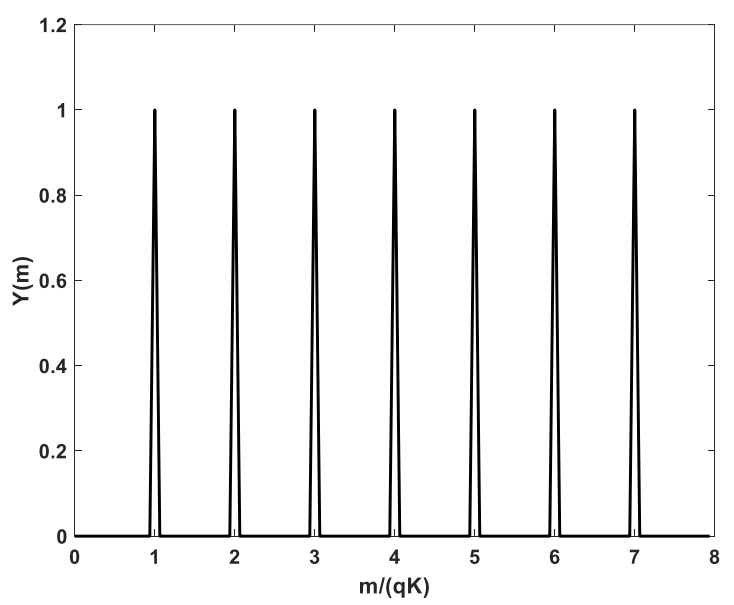

Figure. 4 A reference pattern of the CS

The value of $T h$ is set proportional to the average value of $y(m)$, i.e, $T h=c \bar{y}$ where $c$ is a scaling factor and $y$ is the average value of $y(m)$. This is equivalent to normalizing the metric $y(m)$ in order to have the value of the final decision metric $S$ dependent only on the shape of $y(m)$ and not on the power of $x(n)$ and hence avoiding the noise uncertainty problem caused by error in estimating the noise variance. The decision metric $S$ in Eq. (16) can be viewed as a correlation between the CS metric $y(m)$ and a reference pattern of periodic impulses. If the quantity $q$ is unknown to the CR receiver, Eq. (16) can be evaluated for different values of $q$ and maximizing over $q$, i.e.,

$$
S=\max _{q} \frac{1}{N_{p}} \sum_{k=1}^{N_{p}} y(k q K)
$$

\section{Numerical simulation and results}

The performance of the proposed detection 


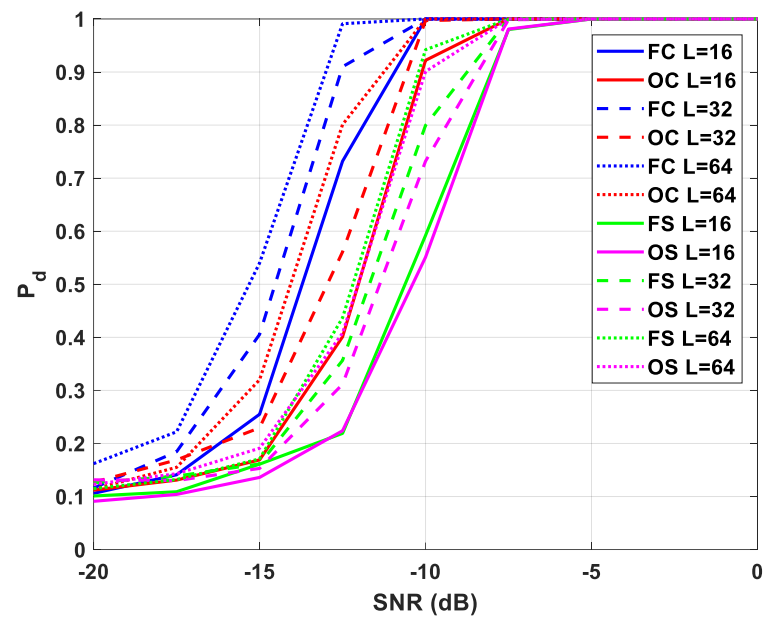

(a)

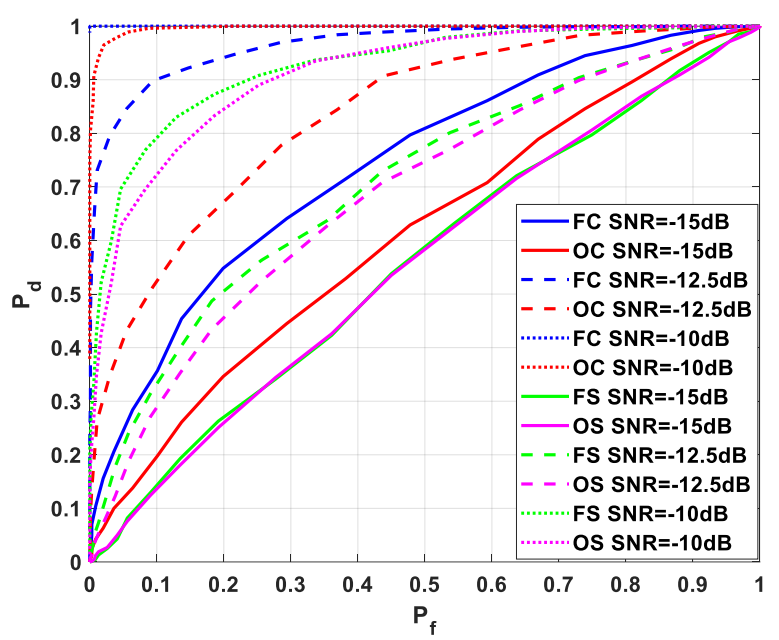

(c)

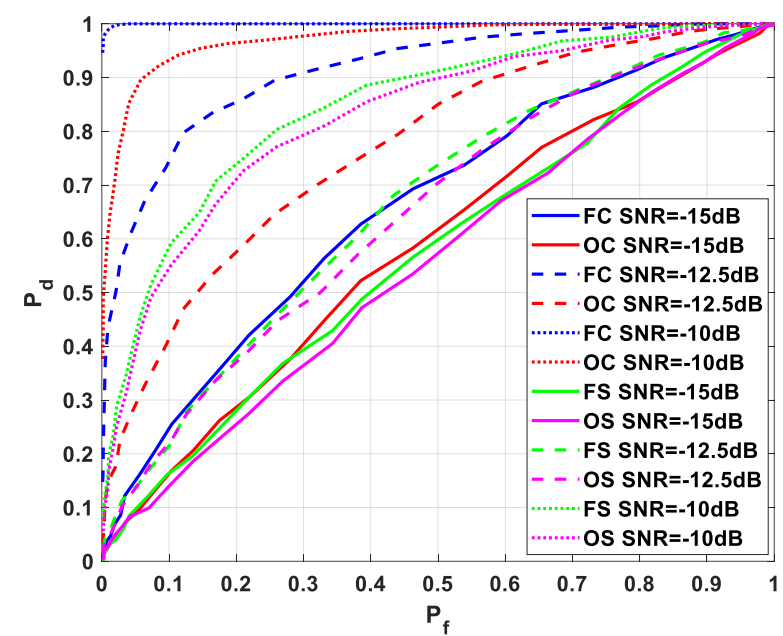

(b)

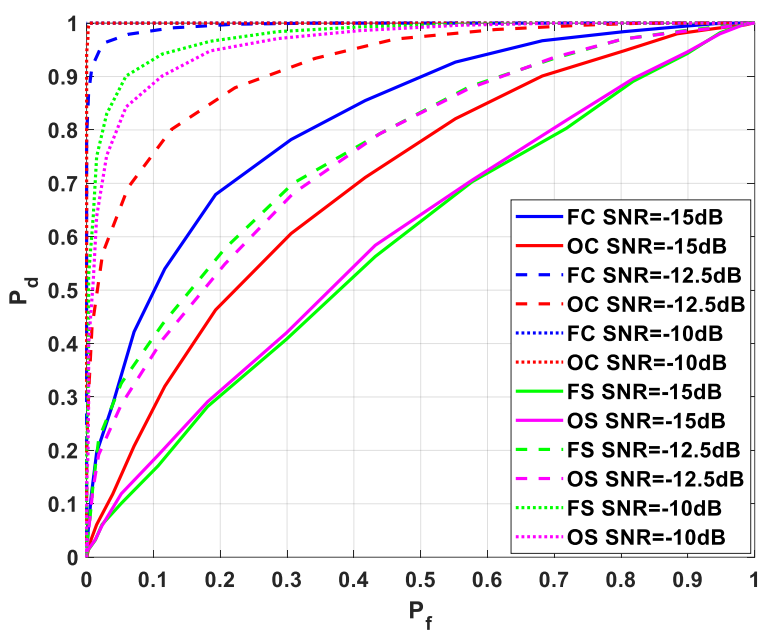

(d)

Figure. 5 Performance under AWGN channel: (a) The $P_{d}$ vs SNR for $P_{f}=0.1$, (b) ROC for L=16, (c) ROC for L=32 (d) ROC for $\mathrm{L}=64,(\mathrm{~F}=\mathrm{FBMC}, \mathrm{O}=\mathrm{OFDM}, \mathrm{S}=$ Scattered and $\mathrm{C}=$ Continuous)

scheme is investigated for detecting OFDM and FBMC signals for different values of $L$ since it affects the complexity and processing time of the detector. The performance is evaluated for both AWGN channel and the multipath fading channel with delay profile of Extended Pedestrian A (EPA) model [24] and Doppler frequency $f_{d}=50 \mathrm{~Hz}$. A summary of the simulation parameters is as below

1. The simulation was performed for number of subcarriers $N=256$, and used subcarriers $N_{u}=180$. The subcarrier bandwidth $F=1 / T_{0}=15 \mathrm{kHz}$ and the $\mathrm{CP}$ time $T_{1}=0.07 T_{0}$; these parameters are chosen to coincide with the LTE parameters [25].

2. The CS is induced by pilots of separation factor of $q=6$ with $p=4$ for scattered pilots and $p=1$ for continuous pilots.

3. The number of the FFT points used to estimate the PSD in Eq. (14), $M$, is chosen to equal the shaping filter length $g(n)$ in FBMC, i.e., $M=K N$ with $K$ typically equals to 4 . The same value of
$M$ is chosen for the PSD estimate for the OFDM case to have a common ground for comparison. The overlap factor $D=N / 2$.

4. The timing offset $n_{o}$ is uniformly distributed over $[0-N-1]$ and the frequency offset is set to $f_{o}=0.1$.

5. The performance is evaluated for different values of segment length, $L$. The values of $N, D$ and $L$ affect the length of the total observation window $\left(T_{w}\right)$ required to estimate the metric $y(m)$

$$
T_{w}=D L+M-D
$$

By substituting $D=N / 2$ and $M=4 N$, Eq. (20) can be written more explicitly in terms of $N$

$$
T_{w}=(L+7) N / 2
$$

The values of $L$ used in the simulation are in the 


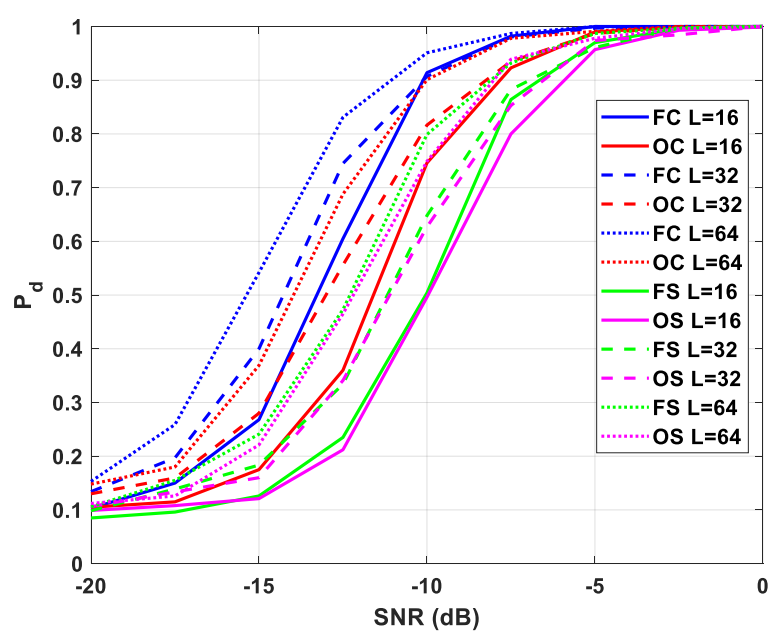

(a)

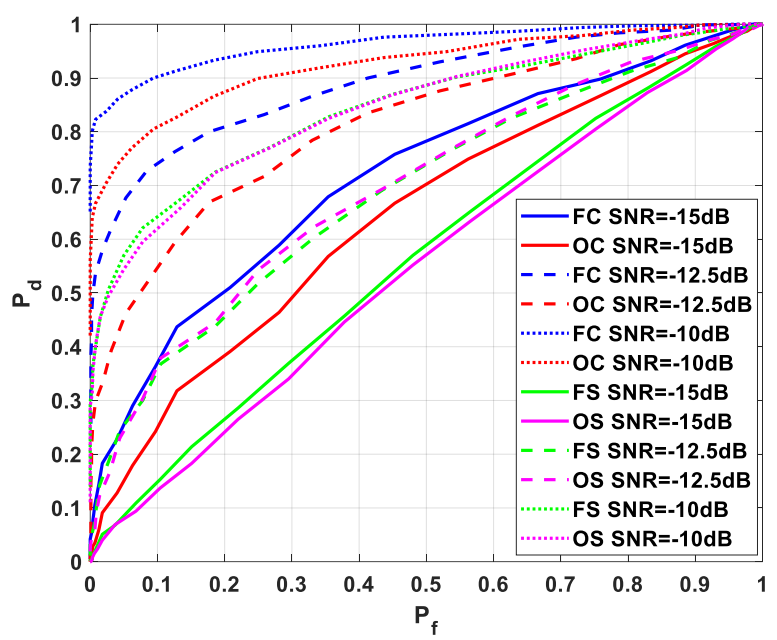

(c)

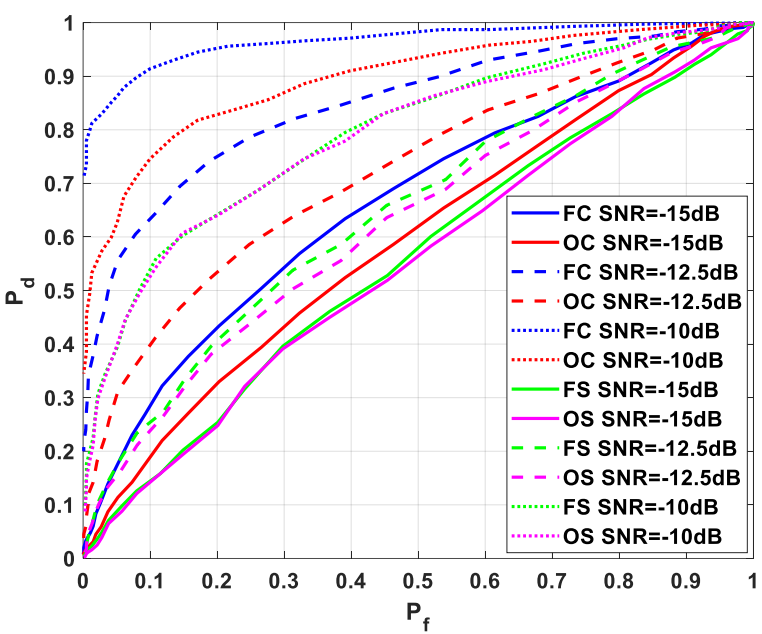

(b)

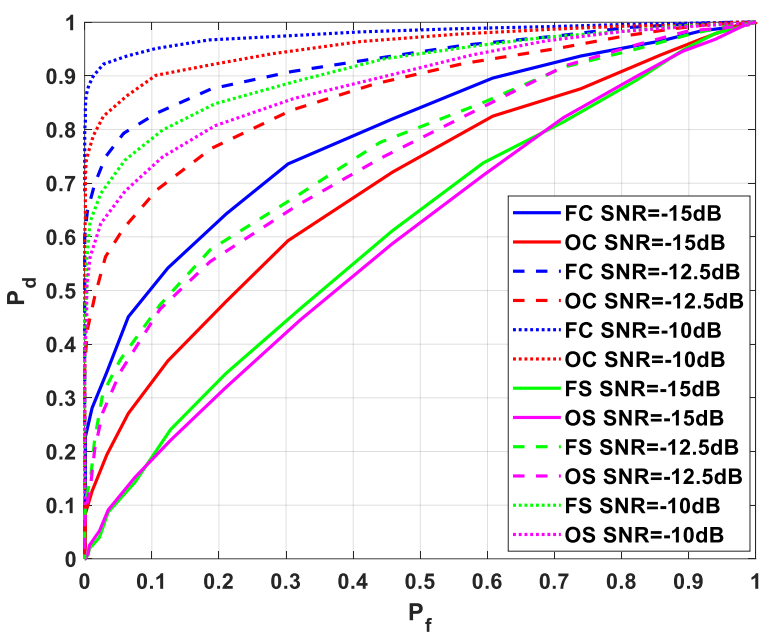

(d)

Figure. 6 Performance under EPA channel: (a) The $P_{d}$ vs SNR for $P_{f}=0.1$, (b) ROC for L=16, (c) ROC for L=32 (d) ROC for $\mathrm{L}=64$, $(\mathrm{F}=\mathrm{FBMC}, \mathrm{O}=\mathrm{OFDM}, \mathrm{S}=$ Scattered and $\mathrm{C}=$ Continuous $)$

range 16-64 making the observation window length in the range $12 \mathrm{~N}-36 \mathrm{~N}$ samples, which is a measure of the processing time. The performance is evaluated in terms of probability of detection $P_{d}$ and probability of false alarm $P_{f}$ under AWGN channel as shown in Fig. 5 and multipath fading channel in Fig. 6. In Fig. 5-a and Fig. 6-a $P_{d}$ vs SNR is shown for $P_{f}=0.1$ and in Fig. 5 (b)-(c) and Fig. 6 (b)-(c) the receiver operating characteristics (ROC) is shown as $P_{d}$ vs $P_{f}$. It is In general, FBMC performs better than OFDM in AWGN and multipath channel in case of continuous pilots while both modulations perform comparably in case of scattered pilots. Since the continuous pilots exhibit stronger CS, there performance is better than the scattered pilots in both OFDM and FBMC. In addition, the detector is robust against lack of synchronization in symbol timing and carrier frequency.

Results show that under AWGN channel, perfect probability of detection, $P_{d}=1$, with $P_{f}=0.1$ can be achieved in the range of $-12 \mathrm{~dB}$ to $-10 \mathrm{~dB}$ SNR. While the same situation is achievable in the range of -7 to $-5 \mathrm{~dB}$ SNR under multipath fading channel depending on the number of frames $L$. Results also show that doubling the value of $L$ results in a 1-2 dB SNR advantage for the probability of detection $P_{d}$. In these simulations, the value of $q$ was assumed to be known to the CR receiver, in case $q$ is unknown and decision metric $\mathrm{S}$ needs to be calculated according to Eq. (19) this means that more computational effort and processing time is required. However, the computations and processing time can be reduced by generating a database at the $\mathrm{CR}$ receiver for each detected PU parameters like the $q$ parameter and the operating frequency. In this case, whenever Eq. (19) is invoked, only the $q$ values stored in the CR's database are used. On the other hand, the main calculations involving the cyclostationary metric calculations in Eqs. (13) and (14) are not affected. 


\section{Conclusions}

A cyclostationary feature detection scheme suitable for OFDM and FBMC signals is presented. The proposed scheme detects cyclostationary features of the signal generated by the inserted pilots. The CS metric is derived as a function of the cyclic frequency from the two-dimensional CAF at zero time lag. This choice of metric offers the advantage of blindly calculating the cyclostationary metric without assuming a prior knowledge of signal parameters needed to calculate the conventional cyclostationary metric at a specific cyclic frequency as in previous works. The CS metric exhibits periodic impulses that represents the cyclostationary feature due to the presence of the pilots. A decision metric is formed as a correlation between CS metric and a reference pattern of impulses generated by the pilots' distribution within the subcarriers, this makes the detection scheme independent of the received signal power. The proposed detection scheme is robust against lack of symbol timing and carrier frequency synchronization between the received PU signal and the $\mathrm{CR}$ receiver. Numerical results show that for continuous pilots the detection scheme has better performance in FBMC over OFDM while for scattered pilots, the performance is almost equal. A trade-off between performance and processing time can be maintained in the choice of the number of processed frames $L$.

\section{Conflicts of Interest}

The authors declare no conflict of interest.

\section{Author Contributions}

Conceptualization, Mohammed Al-Haddad and Hadi Ziboon; methodology, Mohammed Al-Haddad; software, Mohammed Al-Haddad; validation, Mohammed Al-Haddad, and Hadi Ziboon; formal analysis, Mohammed Al-Haddad; investigation, Mohammed Al-Haddad; resources, Mohammed AlHaddad; data curation, Mohammed Al-Haddad; writing - original draft preparation, Mohammed AlHaddad; writing - review and editing, Mohammed Al-Haddad; visualization, Mohammed Al-Haddad; supervision, Hadi Ziboon; project administration, Hadi Ziboon;

\section{References}

[1] T. Yücek and H. Arslan, "A Survey of Spectrum Sensing Algorithms for Cognitive Radio Applications", IEEE Communications Surveys \& Tutorials, Vol. 11, No. 1, pp.161-130, 2009.
[2] K. Maeda, A. Benjebbour, T. Asai, T. Furuno, and T. Ohya, "Recognition Among OFDMBased Systems Utilizing CyclostationarityInducing Transmission", In: Proc. of the 2nd IEEE International Symposium on New Frontiers in Dynamic Spectrum Access Networks, Dublin, Ireland, pp.516-523, 2007.

[3] F. Digham, M. Alouini, and M. Simon, "On The Energy Detection of Unknown Signals Over Fading Channels", IEEE Transactions On Communications, Vol. 55, No. 1, pp.21-24, 2007.

[4] H. Tang, "Some Physical Layer Issues of WideBand Cognitive Radio Systems", IEEE International Symposium on New Frontiers in Dynamic Spectrum Access Networks, Baltimore, Maryland, USA, pp. 151-159, 2005.

[5] N. S. Kim, "Cooperative Overlay Cognitive Radio NOMA Network with Channel Errors and Imperfect SIC", International Journal of Intelligent Engineering and Systems, Vol. 12, No. 5, pp.224-231, 2019.

[6] P. D. Sutton, K. E. Nolan, and L. E. Doyle, "Cyclostationary Signatures in Practical Cognitive Radio Applications", IEEE Journal on Selected Areas in Communications, Vol. 26, No. 1, pp.13-24, 2008.

[7] H. Zhang, D. Le Ruyet, and M. Terre, "Signal Detection for OFDM/OQAM System Using Cyclostationary Signatures", In: Proc. of IEEE 19th International Symposium on Personal, Indoor and Mobile Radio Communications, Cannes, France, pp.131-136, 2008.

[8] P. D. Sutton, J. Lotze, K. E. Nolan, and L. E. Doyle, "Cyclostationary Signature Detection in Multipath Rayleigh Fading Environments", In: Proc. of the 2nd International Conference on Cognitive Radio Oriented Wireless Networks and Communications, Orlando, Florida USA, pp.408-413, 2007.

[9] A. Zahedi-Ghasabeh, A. Tarighat, and B. Daneshrad, "Spectrum Sensing of OFDM Waveforms Using Embedded Pilots in the Presence of Impairments", IEEE Transactions on Vehicular Technology, Vol. 61, No. 3, pp.1208-1221, 2012.

[10] A. A. Thomas and T. Sudha, "Primary User Signal Detection in Cognitive Radio Networks Using Cyclostationary Feature Analysis", In: Proc. of IEEE National Conference on Communication, Signal Processing and Networking (NCCSN), Palakkad, India, pp.1-5, 2014.

[11] A. Kumar and Nandha Kumar P., "OFDM system with cyclostationary feature detection 
spectrum sensing”, ICT Express Vol. 5, No. 1, pp. 21-25, 2019.

[12] L. K. Mathew, A. P. Vinod, and A. S. Madhukumar, "A Cyclic Prefix Assisted Spectrum Sensing Method for Aeronautical Communication Systems", In: Proc. of IEEE International Symposium on Circuits and Systems (ISCAS), Sapporo, Japan, pp.1-5, 2019.

[13] S. Narieda, "Improved MCAS Based Spectrum Sensing in Cognitive Radio", IEICE Transactions on. Communications, Vol. E101-B, No. 3, pp. 915-923, 2018.

[14] S. Narieda, D. Cho, H. Ogasawara, K. Umebayashi, T. Fujii, and H. Naruse, "Derivation of Sensing Features for Maximum Cyclic Autocorrelation Selection Based Signal Detection", In: Proc. of IEEE 90th Vehicular Technology Conference (VTC2019-Fall), Hawaii, USA, pp.1-5, 2019.

[15] P. S. Yawada and A. J. Wei, "Cyclostationary Detection Based on Non-cooperative Spectrum Sensing in Cognitive Radio Network", In: Proc. of IEEE International Conference on Cyber Technology in Automation, Control, and Intelligent Systems (CYBER), Chengdu, China, pp. 184-187, 2017.

[16] K. Kim, I. A. Akbar, K. K. Bae, J. Um, C. M. Spooner, and J. H. Reed, "Cyclostationary Approaches to Signal Detection and Classification in Cognitive Radio", In: Proc. of the 2nd IEEE International Symposium on New Frontiers in Dynamic Spectrum Access Networks, Dublin, Ireland, pp. 212-215, 2007.

[17] K. Upender and S. Chaudhari, "AutocorrelationBased Spectrum Sensing of FBMC Signal", In: Proc. of the 10th International Conference on Communication Systems \& Networks (COMSNETS), Bengaluru, India, pp. 151-158, 2018.

[18] R. Datta, M. Gautier, V. Berg, Y. Futatsugi, M. Ariyoshi, and M. Schühler, "Flexible Multicarrier PHY Design for Cognitive Radio in White Space", In: Proc. of the 6th International ICST Conference on Cognitive Radio Oriented Wireless Networks and Communications (CROWNCOM), Osaka, Japan, pp. 141-145, 2011.

[19] B. Farhang-Boroujeny, "OFDM Versus Filter Bank Multicarrier", IEEE Signal Processing Magazine, Vol. 28, No. 3, pp. 92-112, 2011.

[20] J. Du and S. Signell, "Time Frequency Localization of Pulse Shaping Filters in OFDM/OQAM Systems", In: Proc. of IEEE 6th International Conference on Information,
Communications \& Signal Processing, Singapore, pp. 1-5, 2007.

[21] P. Siohan, C. Siclet, and N. Lacaille, "Analysis and Design of OFDM/OQAM Systems Based on Filter Bank Theory", IEEE Transactions on Signal Processing, Vol. 50, No. 5, pp. 11701183, 2002.

[22] M. Bellanger, "FBMC Physical Layer: A primer", PHYDYAS FP7 Project Document, 2010.

[23] J. G. Proakis and D. K. Manolakis, Digital Signal Processing, 4th Ed. Prentice-Hall, 2006.

[24] 3GPP TS 36.104 (October 2011) Base Station (BS) Radio Transmission and Reception, Release 10, pp.141.

[25] Cox, An Introduction to LTE, John Wiley \& Sons, 2012. 